\title{
An approximate model for the adhesive contact of rough viscoelastic surfaces
}

\author{
Guillaume Haiat ${ }^{1}$ and Etienne Barthel ${ }^{2}$ \\ 1_ CNRS UMR 7052 - B2OA Laboratoire de Mécanique Physique \\ 61, Avenue du Général de Gaulle 94010 Créteil - France \\ 2 Laboratoire CNRS/Saint-Gobain "Surface du Verre et Interfaces", \\ 39, quai Lucien Lefranc, BP 135, F-93303 Aubervilliers Cedex,France.
}

October 29, 2018 


\section{Abstract}

Surface roughness is known to easily suppress the adhesion of elastic surfaces. Here a simple model for the contact of viscoelastic rough surfaces with significant levels of adhesion is presented. This approach is derived from our previous model [E. Barthel and G. Haiat Langmuir, 189362 2002] for the adhesive contact of viscoelastic spheres. For simplicity a simple loading/unloading history (infinitely fast loading and constant pull-out velocity) is assumed. The model provides approximate analytical expressions for the asperity response and exhibits the full viscoelastic adhesive contact phenomenology such as stress relaxation inside the contact zone and creep at the contact edges. Combining this model with a Greenwood-Williamson statistical modeling of rough surfaces, we propose a quantitative assessment of the adhesion to rough viscoelastic surfaces. We show that moderate viscoelasticity efficiently restores adhesion on rough surfaces over a wide dynamic range. 


\section{Introduction}

Surface roughness is a major parameter for the control of the adhesion of viscoelastic materials and as such is central to many technological processes. As an example, in the automotive industry, when assembling a polymer interlayer with two glass sheets to form a laminated windshield, a periodic roughness is intentionally generated on the polymer to reduce the adhesion and facilitate positioning before the final heat treatment [1]. Roughness also plays a role in the self-adhesion mechanism (or tack) of soft polymers [2, 3] like repositionable adhesives. In a similar way, in the glass industry, glass molding is performed at elevated temperatures for easy glass flow but at the risk of adhesion. Indeed adhesion of hot glass to the mold halts production until the mold has been cleaned of its adherents. The roughness of the mold strongly impacts the adhesion of the viscoelastic glass gob [4] and a stochastic but carefully tailored roughness, obtained through shot peening of the steel surfaces for instance, will promote an easy molding process.

The adhesive contact to rough viscoelastic surfaces is a complex problem however because it couples the statistical approach of the adhesive contact to rough surfaces with the difficulties involved in the viscoelastic adhesive contact. For elastic solids, the adhesive contact to rough surfaces is reasonably well understood. Building upon the Greenwood-Williamson independent asperity approach, Fuller and Tabor [5] have given a useful description of the strong reduction of the adhesive force incurred when roughness increases. They introduce an adhesion parameter $\sigma_{s} / \delta_{c}$ where $\sigma_{s}$ is the standard deviation of the summit height distribution and $\delta_{c}$ is the maximum single asperity extension before rupture. This adhesion parameter is a measure of the energy balance between stretched and compressed asperities. If the adhesion parameter is large, the compression of the upper asperities dominates the traction of the adhesive lower asperities which significantly depresses the adhesion.

The calculations are relatively straightforward for elastic contacts which are essentially reversible. For viscoelastic contacts, adhesion depends upon the full contact history so that the calculations are more involved. In the absence of adhesion, the viscoelastic contact problem was first solved by Ting [6]. Subsequently, important results for the viscoelastic crack problem were obtained by Schapery [7, 8] and Greenwood and Johnson [9, 10]. Hui and coworkers [11, 12, 13] have tackled the problem of viscoelastic adhesive contacts. We recently proposed a model for the full viscoelastic contact of adhesive bodies [14, 15, 16].

The present paper inquires into the application of this model to the adhesive contact to rough surfaces. To model the response of a distribution of asperities a simplified description of the viscoelastic contact is needed and the first part of the paper develops such a minimal model. It is devised so that the two main phenomena characteristic for viscoelastic adhesive contact - stress relaxation under the contact and creep induced by the adhesive interactions at the contact 
edges - are preserved while keeping calculations to a minimum. The model exhibits typical viscoelastic adhesive contact phenomenology, and in particular the time lag between indenter retraction and the actual contact radius recession, also called "stick period" [14. In the second part of the paper examples of the use of this approximate model for rough surfaces are presented. The impact of the pull-out velocity on the pull-out force for typical rough surfaces is calculated. The results emphasize how a moderately viscoelastic material effectively restores the adhesion lost through roughness.

\section{Model}

\subsection{Single asperity contact}

Modeling the viscoelastic contact of an axisymmetric asperity in the presence of adhesion is complex because, as in all viscoelastic problems, the solution depends upon the full contact history.

In addition, for contact problems, one must deal with mixed boundary conditions: the surface normal displacement $u(r)$ is prescribed inside the contact zone whereas the normal surface stress $\sigma(r)$ due to the adhesive interactions is specified inside the so-called cohesive zone (Figure 1), outside the contact zone. Moreover the boundary between these two regions is not fixed but moves during the contact history. For a viscoelastic material, the solution is reasonably simple for growing contact radius [11, 17. However, during pull-out (i.e. decreasing contact area), the residual deformation has to be taken into account. This in turn depends upon the full stress history, which leads to a complex situation, both for adhesionless [6] and adhesive [11, 12, 13, 14, 15] contacts.

\section{$2.2 \quad$ Resolution method}

Under the assumption of axial symmetry, we resort to two auxiliary functions $g(r)$ and $\theta(r)$, which are the following Abel transforms of respectively the distributions of normal surface stress $\sigma(r)$ and normal surface displacement $u(r)$ :

$$
\begin{aligned}
& g(r)=-\int_{r}^{+\infty} \frac{s \sigma(s)}{\sqrt{s^{2}-r^{2}}} d s, \\
& \theta(r)=\frac{d}{d r} \int_{0}^{r} \frac{s u(s)}{\sqrt{r^{2}-s^{2}}} d s,
\end{aligned}
$$

\subsubsection{Boundary conditions}

A first benefit of these transforms is their pertinence for axisymmetric boundary conditions. In particular, inside the contact zone $(r<a)$, the normal surface 
displacement is known through the contact condition

$$
u(r)=\delta-h(r)
$$

where $\delta$ is the penetration and $h(r)$ the shape of the indenter (Figure 1). Then, using Eq. 2, inside the contact zone

$$
\theta(r)=\delta-\delta_{0}(r)
$$

where

$$
\delta_{0}(r)=\frac{d}{d r} \int_{0}^{r} d s \frac{s h(s)}{\sqrt{r^{2}-s^{2}}} .
$$

It turns out that $\delta_{0}(r)$ is the Hertz penetration for a contact radius $r$ as shown in the next section. Note that this function $\delta_{0}(r)$ is determined by the shape of the indenter only. It is equal to $r^{2} / R$ for a sphere of radius $R$ and to $\frac{\pi}{2} r / \tan \beta$ for a cone of apical angle $\beta$.

Conversely, as mentioned above, the adhesive stress distribution $\sigma(r)$ outside the contact zone is known. More precisely, in the present case, we will show in section 2.2 .3 that it can be self-consistently determined through an independent set of equations, under the assumption of small cohesive zone size. Therefore the $g$ function is known outside the contact zone $(r>a)$.

\subsubsection{Mechanical Equilibrium - Viscoelastic materials}

The second benefit of these auxiliary functions is that they naturally handle the long range nature of the elastic field: for instance, under the conditions of linear elastic behaviour (Young's modulus E, Poisson ratio $\nu$ ) and absence of shear stresses at the contact, mechanical equilibrium leads to

$$
g(r)=\frac{E^{\star}}{2} \theta(r)
$$

where

$$
E^{\star}=\frac{E}{\left(1-\nu^{2}\right)}
$$

The contact problem is solved under the assumption of continuity of the stress distribution at $a$. The penetration is then directly obtained by [14]

$$
g(a)=\frac{E^{\star}}{2} \theta(a)
$$

For example for an adhesionless contact, $\sigma(r)=0$ for $r \geq a$ so that $g(a)=0$ and $\delta=\delta_{0}(a)$ as mentioned in section 2.2.1. If adhesion is present, $g(a)$ is not zero and a more complex situation arises. 
For a linear viscoelastic material, under the approximation of constant Poisson ratio [18] we introduce the creep and the relaxation functions

$$
\begin{aligned}
\phi(t) & =\frac{2}{E^{*}} \tilde{\phi}(t) \\
\psi(t) & =\frac{E^{*}}{2} \tilde{\psi}(t)
\end{aligned}
$$

with

$$
\begin{aligned}
\tilde{\phi}(t) & =1+\frac{(1-k)}{k}\left(1-e^{\frac{-t}{\mu}}\right) \\
\tilde{\psi}(t) & =k+(1-k) e^{\frac{-t}{k \mu}}
\end{aligned}
$$

where $\mu$ is the creep time. The parameter $k$ lies between 1 (elastic) and 0 (Maxwell). Due to the decoupling between spatial and temporal responses, the equilibrium equation (Eq. 6) will now be

$$
g(r, t)=\int_{0}^{t} d \tau \psi(t-\tau) \frac{d}{d \tau} \theta(r, \tau)
$$

or its inverse

$$
\theta(r, t)=\int_{0}^{t} d \tau \phi(t-\tau) \frac{d}{d \tau} g(r, \tau)
$$

where the relaxation function $\psi(t)$ and the creep function $\phi(t)$ are inverse for this product of convolution. In the case of increasing contact radius, Eqs. 4 and 14 determine the penetration by

$$
\delta(t)=\delta_{0}(a(t))+\int_{0}^{t} d \tau \phi(t-\tau) \frac{\partial}{\partial \tau} g(a(t), \tau)
$$

This result is equivalent to previous formulations [12, 17].

The decreasing contact radius case is more intricate. Taking into account the domains where $g$ and $\theta$ are known respectively, we have to resort to Eqs. 4, 13 and 14 to determine the penetration by the integral equation

$$
g(a(t), t)=\int_{t_{a-}(a(t))}^{t} d \tau \psi(t-\tau) \frac{\partial}{\partial \tau}\left\{\delta(\tau)-\delta_{0}(a(t))\right\}+\bar{g}(a(t), t)
$$

Here $t_{a-}(a(t))$ is the time at which the contact radius was first equal to $a(t)$, during the loading phase. The corrective term

$$
\bar{g}(a(t), t)=\int_{0}^{t_{a-}(a(t))} d \tau \psi(t-\tau) \frac{\partial}{\partial \tau}\left(\int_{0}^{\tau} d \tau^{\prime} \phi\left(\tau-\tau^{\prime}\right) \frac{\partial}{\partial \tau^{\prime}} g\left(a(t), \tau^{\prime}\right)\right)
$$

is unessential, except for the cross-over between increasing and decreasing contact radius regimes $\left(a=a_{\max }, t=t_{\max }\right)$. 
Then for a given history of the penetration, one can calculate the history of the contact radius and subsequently the force [14, 15, 16]. Under the assumption of small cohesive zone size (see section 2.2.3 for the cohesive zone and section 6 for details of the calculation) the force is given by Eq. 19 in [14]:

$$
P(t)=4 \int_{0}^{t} d \tau \psi(t-\tau) \frac{d}{d \tau} \int_{0}^{\min (a(t), a(\tau))} d r\left(\delta(\tau)-\delta_{0}(r)\right)
$$

Note that this equation is valid for both inward and outward runs. Eqs. 16 and 18 involve stress relaxation inside the contact zone but also crack tip viscoelasticity through $g(a(t), t)$, as discussed in more details in the next paragraph.

\subsubsection{Cohesive Zone}

For any adhesive contact, in the context of the present (Sneddon) method, the quantity $g(a)$, which is the function $g$ evaluated at the edge of the contact zone, plays a central role. For the contact zone, it determines Eqs. 15, 16] and 18 between macroscopic contact variables. But we have previously shown that, for the cohesive zone, $g(a)$ determines the stress intensity factor [19] characteristic of the local deformation process at the crack tip.

Here we dicuss, for the specific case of a viscoelastic adhesive contact, how $g(a(t), t)$ is determined by the cohesive zone parameters. This results in an independent relation between $g(a)$ and the contact radius velocity $\dot{a}$ through Eqs. 20 , 21, 22 and 23.

This problem is similar to a viscoelastic crack [7, 8, 9, 10] and calls for a consistent treatment of an adhesive-stress induced creep inside a cohesive zone of finite size $(c(t) \geq r \geq a(t)$, Figure 1). The equivalence of various methods for the description of the cohesive zone has been demonstrated earlier [20]. In brief, the details of the description of the stresses inside the cohesive zone is unimportant as long as the effective range of these interactions is preserved. Greenwood and Johnson [21] have proposed a description (the so-called "Double-Hertz") which is analytically simple and which we have retained for our viscoelastic models [14]. Following these authors, we assume a specific stress distribution which results in

$$
g(r)=\frac{\pi}{4} \sigma_{0} \frac{r^{2}-c^{2}}{\sqrt{c^{2}-a^{2}}}
$$

Assuming that the size of the cohesive zone is small compared to the contact zone $(a \gg c-a \equiv \epsilon)$ and taking the limit when $r \rightarrow a$ gives:

$$
g(a)=-\frac{\pi}{4} \sigma_{0} \sqrt{2 a \epsilon}
$$

For a decreasing contact radius the self-consistent equation coupling the total adhesion energy and the deformation of the contact zone writes (Eq. 23 of Ref. [14]):

$$
w=\frac{\pi}{8} \sigma_{0}^{2} \epsilon \phi_{1}\left(t_{r}\right)
$$


where $w$ is the adhesion energy,

$$
\phi_{1}(t)=\frac{2}{t^{2}} \int_{0}^{t} d \tau \tau \phi(t-\tau)
$$

and $t_{r}$ is a fracture dwell time. For non vanishing contact radius velocity, this is the time it takes for the crack tip to move a distance equal to the cohesive zone size. It is given by:

$$
t_{r}(t)=\frac{-\epsilon(t)}{\dot{a}(t)}
$$

The simultaneous resolution of Eqs. 20-23 determines the velocity dependence of $g(a)$. It is typical of viscoelastic crack problems : compare our set of Eqs. 20, 21 and 23 with, for instance, Eqs. 1, 47 and 48 in [7. As a result, $g(a)$ increases with $\dot{a}$ as displayed on Figure 2, again compare e. g. with Fig. 6 b in [10].

\subsubsection{General Model - stick period}

For the viscoelastic adhesive contact, the main phenomenon we have identified [14, 15] is the "pinning" of the contact upon unloading (Figure 3). More precisely, for such viscoelastic contacts, there is a typical time lag between the retraction of the indenter and the actual contact radius recession. We have called "stick period" this period over which the contact radius stays pinned, before contact edge retraction sets in, finally leading to contact rupture.

The physical interpretation of the stick period phenomenon has already been given in [14]. Relaxation of the stresses inside the contact zone reduces the stress intensity factor and hence $g(a)$. This results in a slow crack motion (Figure 2) and the contact radius practically comes to a halt. When pulling the indenter out, enough tensile stresses must be re-built inside the contact zone before $g(a)$ is large enough for the contact edge to be restored into propagating condition (Figure 2), and this depends upon the competition between stress relaxation and pull out velocity as discussed in [16].

\subsection{Approximate Model}

We need a simple description of the viscoelastic adhesive contact for rough surface calculations. We restrict the developments to the spherical asperity (radius $R$ ) usual in rough surface models although extension to other asperity shapes should be straightformard. We also introduce two major simplifications to the full model. One is an assumption on the type of loading/unloading. The other one is an approximation for the stick period.

\subsubsection{Simplifying assumption - Direct unloading}

We consider fixed-grip loading, i.e. a loading where the penetration is prescribed. In addition, the loading phase is considered to be infinitely fast and the pull-out 
velocity $\dot{\delta}$ a (negative) constant (Figure 3), so that the penetration history simply writes:

$$
\delta(t)=\left(\delta_{0}+t \dot{\delta}\right) \Theta(t)
$$

where $\delta_{0}$ is the initial penetration 1 , taken positive when the two surfaces are pushed one into another. $\Theta$ is the Heaviside step function. Due to the infinitely fast loading phase the quantity $t_{a-}(a(t))$ defined in the unloading phase (section 2.2.2) is equal to zero.

\subsubsection{Stick period approximation}

This approximation stems from the observation that for large penetrations, the contact radius upon loading is primarily determined by the instantaneous elastic response and that crack tip creep during loading is not a dominant process [14].

As a result, in the following, the stick period is approximately modeled by a period of constant contact radius prescribed by the instantaneous modulus. This will be a good approximation except for small initial contact radii where the adhesive term is dominant.

In summary the approximate model is as follows:

1. calculate the initial contact radius from an elastic model with the instantaneous Young's modulus; assume this contact radius stays constant to the end of the stick period. The main viscoelastic process at work here is the stress relaxation inside the contact zone, which directly impacts the force (section 2.3.3);

2. determine if there is a stick period and if so calculate ending time $t_{f}$ (section 2.3.4);

3. calculate the unloading phase (section 2.3.5) and in particular the adhesion force.

\subsubsection{Stick Period - Force}

The force in the stick period is obtained from Eq. 18 taking into account the discontinuity of the penetration at $\tau=0$. This leads to (section 6.1):

$$
P_{1}(t)=4 \psi(t)\left(\frac{2 a_{0}^{3}}{3 R}-\frac{\sigma_{0} \pi a_{0}^{3 / 2} \sqrt{\epsilon_{0}}}{\sqrt{2} E^{*}}\right)+4 a_{0} \dot{\delta} \int_{0}^{t} d \tau \psi(t-\tau)
$$

which can be rewritten as:

$$
P_{1}(t)=\tilde{\psi}(t) P_{0}+4 a_{0} \dot{\delta} \int_{0}^{t} d \tau \psi(t-\tau)
$$

\footnotetext{
1 This initial penetration cannot be confused with the $\delta_{0}(r)$ function introduced earlier and which will now be substituted by its specific value for a sphere $r^{2} / R$ in the rest of the paper.
} 
where the normalized form of $\psi$ (Eq. 12) has been used. $P_{0}$ is the initial force and $\epsilon_{0}$ the cohesive zone size at $t=0$ which can be calculated from Eqs. 20, 21, The first term results from the relaxation of the initial force and the second one is due to the flat punch viscoelastic response and is proportional to the (constant) pull-out velocity.

\subsubsection{Approximate determination of the end of the stick period}

At the end of the stick period $a\left(t_{f}\right)=a_{0}$ because the contact radius increase has been neglected by assumption. However we do take into account the crack tip evolution which is reflected in a cohesive zone size $\epsilon\left(t_{f}\right)$ smaller than $\epsilon_{0}$. Since the contact radius is a constant, the crack dwell time $t_{r}$ (section 2.2.3) is approximated as the crap tip age, which is also the contact age. Therefore, when the contact radius starts decreasing at $t=t_{f}$, the dwell time $t_{r}$ is taken equal to $t_{f}$. Inserting Eq. 21 into Eq. 20 provides an approximate criterion for the begining of the contact radius decrease:

$$
g\left(a\left(t_{f}\right), t_{f}\right)=-\sqrt{\frac{w \pi a_{0}}{\phi_{1}\left(t_{f}\right)}}
$$

When the contact radius decreases, Eq. 16 becomes

$$
g(a(t), t)=\dot{\delta} \int_{0}^{t} d \tau \psi(t-\tau)+\psi(t)\left(\delta_{0}-\frac{a(t)^{2}}{R}\right)
$$

Then $t_{f}$ is solution to the following implicit equation:

$$
\psi\left(t_{f}\right)\left(\delta_{0}-\frac{a_{0}^{2}}{R}\right)+\dot{\delta} \int_{0}^{t_{f}} d \tau \psi\left(t_{f}-\tau\right)+\sqrt{\frac{w \pi a_{0}}{\phi_{1}\left(t_{f}\right)}}=0
$$

Note that the purely elastic case is recovered for $t=0$. It is also recovered for constant $\psi(t)$ (elastic material) because $\delta(t)=\delta_{0}+\dot{\delta} t$, and for long waiting times where $\psi(t) \simeq \psi(+\infty)$ and $\int_{0}^{t} \psi(\tau) d \tau \simeq t \psi(+\infty)$.

Injecting the initial elastic solution, $t_{f}$ is identified as the non zero root of function $f$, with

$$
f(t)=\dot{\delta} \int_{0}^{t} d \tau \psi(t-\tau)+\sqrt{w \pi a_{0}}\left(\frac{1}{\sqrt{\phi_{1}(t)}}-\psi(t) \sqrt{\frac{2}{E^{*}}}\right)
$$

Time $t_{f}$ only depends on the mechanical properties of the material and on the history of the penetration $\left(\delta_{0}\right.$ and $\left.\dot{\delta}\right)$. Considering a given contact experiment on a rough surface, the mechanical and adhesive properties as well as the pull-out velocity are identical for all asperities. Therefore, $t_{f}$ will only be a function of the initial penetration $\delta_{0}$ which is depends on the initial height of a given asperity. 
The numerical calculation of $t_{f}\left(\delta_{0}\right)$ is achieved using a bisection method. From Eq. [30, and knowing that $a_{0}$ increases with $\delta_{0}, t_{f}\left(\delta_{0}\right)$ is a strictly increasing function of $\delta_{0}$ and can thus be numerically inverted to obtain the function $z_{f}(t)$, representing the initial penetration necessary for a given asperity to reach the end of the stick period at time $t$. This function will be useful for the computation of the total force contribution (section 2.5) from the asperity height distribution.

\subsubsection{Decreasing contact radius}

The computation of the load $P_{2}(t)$ in the case of a decreasing contact radius is performed using Eq. 18 which leads (section 6.2) to:

$$
P_{2}(t)=4 a(t) \psi(t)\left(\delta_{0}-\frac{a(t)^{2}}{3 R}\right)+4 a(t) \dot{\delta} \int_{0}^{t} d \tau \psi(t-\tau)
$$

The two terms are respectively the elastic force for a spherical asperity with the relaxed modulus at time $t$ in presence of adhesion and the response of the viscoelastic medium to a flat punch displacement (contact radius $a(t)$ ). Note that the purely elastic case is recovered for elastic materials because $\delta(t)=\delta_{0}+\dot{\delta} t$. Eqs. 21, 23, 28 and 31 completely describe the decreasing contact radius phase.

\subsection{Single Asperity - Normalization}

For a spherical asperity, following Maugis [14, 22, the normalization of the contact variables is performed by introducing the following quantities:

$$
\begin{aligned}
\tilde{P} & =\frac{P}{\pi w R}, \\
A & =\frac{a}{\left(\frac{3 \pi w R^{2}}{4 E^{\star}}\right)^{1 / 3}}, \\
\tilde{\delta} & =\frac{\delta}{\left(\frac{9 \pi^{2} w^{2} R}{16 E^{2}}\right)^{1 / 3}}, \\
\lambda & =\frac{2 \sigma_{0}}{\left(\frac{\pi w 16 E^{\star 2}}{9 R}\right)^{1 / 3}}, \\
G(A) & =-\frac{\pi}{3} \lambda \sqrt{2 A \tilde{\epsilon}} .
\end{aligned}
$$

were $\tilde{\epsilon}$ is the normalized size of the cohesive zone (same length scale as contact radius $a$ ). In normalized form, $g(a)$ is given by:

$$
G\left(A_{0}\right)=-\frac{\pi}{3} \lambda \sqrt{2 A_{0} \tilde{\epsilon}_{0}}
$$


In normalized form, the self-consistent equation 21 is given by:

$$
1=\frac{\pi^{2}}{12} \lambda^{2} \tilde{\epsilon}(t) \tilde{\phi}_{1}(\operatorname{tr}(t))
$$

where $\tilde{\phi}_{1}$ is given by:

$$
\tilde{\phi}_{1}\left(t_{r}\right)=\frac{2}{t_{r}{ }^{2}} \int_{0}^{t_{r}} d \tau(\tau) \tilde{\phi}(\tau)
$$

Consequently, Eq. 29] used for the determination of the end of the first regime now writes:

$$
\tilde{\psi}(t)\left(\tilde{\delta}(0)-A_{0}^{2}\right)+\dot{\tilde{\delta}} \int_{0}^{t_{f}} d \tau \tilde{\psi}\left(t_{f}-\tau\right)+2 \sqrt{\frac{2 A}{3 \tilde{\phi}_{1}\left(t_{f}\right)}}=0
$$

In the stick period, the force for one isolated asperity (Eq. 18) is now given by:

$$
\tilde{P}_{1}(t)=\tilde{\psi}(t)\left(A_{0}^{3}(t)-A_{0}^{1.5} \sqrt{6}\right)+\frac{3}{2} A_{0} \dot{\tilde{\delta}} \int_{0}^{t} d \tau \tilde{\psi}(t-\tau)
$$

When the contact radius starts decreasing, $G(A(t))$ (Eq. 28) writes as follow :

$$
G(A(t))=\dot{\tilde{\delta}} \int_{0}^{t} d \tau \tilde{\psi}(t-\tau)+\tilde{\psi}(t)\left(\tilde{\delta}(0)-A^{2}(t)\right)
$$

and the force in the second regime is expressed through:

$$
\tilde{P}_{2}(t)=\tilde{\psi}(t)\left(\frac{3}{2} A(t) \tilde{\delta}_{0}-\frac{A^{3}(t)}{2}\right)+\frac{3}{2} A(t) \dot{\tilde{\delta}} \int_{0}^{t} d \tau \tilde{\psi}(t-\tau)
$$

\subsection{Accounting for a rough surface}

We follow the roughness description proposed by Greenwood and Willamson [23] in their adhesionless elastic contact study. Namely, a surface of nominal area $A_{0}$ with $N$ asperities is in contact with a rigid flat plane. All asperities are assumed to have the same radius of curvature $R$ and a Gaussian height distribution of standard deviation $\sigma_{s}$ :

$$
\chi(z)=\frac{1}{\sigma_{s} \sqrt{2 \pi}} \exp \left(-\frac{z^{2}}{2 \sigma_{s}^{2}}\right)
$$

where $\chi(z)$ is the probability that the summit of an asperity stands between $\mathrm{z}$ and $z+d z$. For a penetration of the rigid flat $\delta_{0}$, the number of asperities in contact is given by: $n=N \int_{\delta_{0}}^{\infty} d z \chi(z)$ and all contact variables can be obtained with the same integration. Similarly, the calculation of the load at time $t$ is performed by integrating the viscoelastic response of each asperity initially in contact:

$$
P(t)=N \int_{0}^{\infty} d z \chi\left(z+\delta_{0}\right) P_{a}(z, t)
$$

where $P_{a}(z, t)$ is the load at time $t$ of an asperity undergoing an initial penetration of $z-\delta_{0}$. 


\section{Results and discussion}

\subsection{Single asperity - Impact of pull-out velocity}

Force $v s$ penetration curves (force plots) for a single asperity were calculated with $\lambda=5-$ a value consistent with our assumption of small but finite cohesive zone size - and for a moderately viscoelastic material with $k=0.5$. Figures 4 and 5 display results obtained for $\tilde{\delta}_{0}=5$ for pull-out velocities $\tilde{\dot{\delta}}$ ranging from -0.01 to -50 .

For very low pull-out velocity values $(|\tilde{\dot{\delta}}| \leq 0.1)$, there is an initial rapid decrease of the force, followed by a linear variation of the force as a function of penetration. This is due to the fast relaxation of the (predominantly compressive) stresses. The stress field is then controlled by the relaxed modulus which leads to constant contact stiffness and a linear force-distance curve segment, before the contact radius starts decreasing. When the penetration decreases further, the force becomes tensile (negative), displays an incurvation, levels off, finally increases and jumps to zero: this evolution of the force results from contact edge recession and asperity snap-off. The absolute value of the maximum tensile force defines the pull-out force $F_{\text {pullout }}$, and the (positive) effective adhesion $w_{\text {eff }}$ through $w_{\text {eff }}=F_{\text {pullout }} /(3 / 2 \pi R)$.

The very low velocity case is an elastic adhesive contact with an effective modulus equal to the relaxed modulus and a pull-out force equal to $3 / 2$ which is the JKR value. At slightly larger pull-out velocities, we observe that the pull out force increases with pull-out velocity from this $3 / 2$ value: higher pull-out velocity leads to increased contact radius velocity. Enhancement of the viscoelastic crack tip dissipation and effective adhesion follow as amply demonstrated in the literature [7, 8, 9, 11, 12, 17, 24]. It results from the combination of a fully relaxed contact zone and a less relaxed cohesive zone.

In contrast, for large pull-out velocities $(|\tilde{\dot{\delta}}| \geq 1)$, the initial precipitous drop of the force is no longer present and the pull-out force decreases with pull-out velocity. Eventually for very large pull-out velocity $($ e.g. $\tilde{\dot{\delta}}=-50)$ the behavior tends toward an elastic behavior and the pull out force reverts to $3 / 2$. Indeed, for large pull-out velocities, the relaxation of the compressive stresses in the contact zone is far from complete during the experimental time. The pull-out force is lower than for low pull-out velocity because the compressive contact stresses favor contact rupture.

As a consequence, there exists a transition with an optimum pull-out velocity (here of around $\tilde{\dot{\delta}}=-1$ ) for which the pull-out force is maximum. This result specifically originates from the consistent inclusion of both viscoelastic crack tip dissipation and contact stress relaxation. 


\subsection{Rough surfaces}

As an example of results for rough surfaces, the same material parameters $\lambda=5$ and $k=0.5$ are chosen. The initial penetration is $\tilde{\delta}_{0}=5$.

\subsubsection{Force distribution}

Normalizing the roughness as the penetration (Eq. 34), we compute the force curve for a rough surface with a normalized standard deviation $\tilde{\sigma}_{s}=2$. Computing the contributions of asperities displaying each regime (stick period vs. decreasing contact radius) to the total force for pull-out velocity $\tilde{\dot{\delta}}=-1$ (Figure 6), we observe that the proportion of asperities initially in contact is almost $100 \%$, as expected for such a ratio of initial penetration $\tilde{\delta}_{0}$ to roughness $\tilde{\sigma}_{s}$. This type of contact is relevant to glass molding where a rather homogeneous distribution of asperities, with narrow asperity height and radius distributions, is generated on the mold surface. Note that the Greenwood-Williamson model is particularly apt at treating such a surface morphology. It would be less adequate for a less regular surface with a wide distribution of asperity height and/or a wide distribution of asperity radius.

The force is initially dominated by asperities pinned in the stick period (black dashed line). A gradual transition where the contact zones of the lower asperities de-pin and start to recede is observed: the contribution of pinned asperities drops to zero. Then the lowest asperities start to undergo contact rupture. It is in the middle of this transition that the maximum tensile force (pull-out force) is recorded.

\subsubsection{Impact of roughness - Constant pull-out velocity}

Force plots with increasing roughness (from $\tilde{\sigma}_{s}=0.1$ to $\tilde{\sigma}_{s}=5$ ) are displayed on Figure 7. For small $\tilde{\sigma}_{s}$, the results track the single asperity limit: all the asperities in the population exhibit an identical behaviour, having nearly the same initial penetration. For larger $\tilde{\sigma}_{s}$, the initial contact force increases as expected from the elastic (JKR) limit: for a single asperity the increase of the force with initial penetration is more rapid than linear. Simultaneously, the duration of the contact increases and the pull-out force decreases with roughness as in the elastic case [5]. Indeed, for one isolated asperity, the pull-out time increases with initial penetration. Therefore, when $\tilde{\sigma}_{s}$ increases, the distribution of the pull-out times spreads out and the different asperities contribute less constructively to the total pull-out force, which therefore decreases.

\subsubsection{Impact of pull-out velocity - Constant roughness}

Figures 8 and 9 display force plots at different pull-out velocity values, from $\tilde{\dot{\delta}}=-0.01$ to $\tilde{\dot{\delta}}=-10$ for a normalized standard deviation $\tilde{\sigma}_{s}=2$. The overall 
picture is similar to the single asperity case (section 3.1) and the same transition from low to high velocity regimes is observed. At low pull-out velocities, a fast decrease of the force due to stress relaxation is followed by a quasi elastic behavior while at large velocities, the system is purely elastic. The pull-out forces in these two limit regimes are differentiated by the roughness. This is because the elastic moduli involved are different: indeed, the relaxed modulus is pertinent for the very low pull-out velocity regime, and the instantaneous modulus for the very high velocity regime. A larger elastic modulus will result in a larger adhesion parameter and a lower adhesion force [5, 25]. A pull-out force enhancement is recorded between these two limit regimes because of the combination of partial contact stress relaxation and crack tip dissipation.

It is interesting to assess the details of the transition between these two regimes. Calculation of pull-out forces as a function of pull-out velocities were performed for a rough surface with a roughness of $\tilde{\sigma}_{s}=2$, a cohesive zone parameter $\lambda=5$ and a moderately viscoelastic material $k=0.5$. The pull-out force as a function of pull-out velocity has been normalized to the rough surface limit in the high velocity elastic regime (instantaneous modulus). The results are displayed on Fig. 10 where the isolated asperity result is also shown for comparison. As expected both exhibit enhancement of the pull-out force in the intermediate regime where contact stress relaxation and crack tip creep occur at the same time. Figure 10 clearly evidences that the enhancement of adhesion is much more pronounced on rough viscoelastic surfaces than for an isolated asperity. Indeed for elastic materials a roughness as large as $\tilde{\sigma}_{s}=2$ severely depresses the pull-out force due to the stored elastic energy of the higher asperities. However for a viscoelastic material these elastic stresses rapidly relax while the single asperity adhesive force is enhanced through crack tip dissipation. As a result, even a very moderately viscoelastic material is sufficient to enhance the effective adhesion on rough surfaces by an order of magnitude over a large range of pull out forces (Figure 101).

\section{Conclusion}

A simple model for the adhesive contact of rough viscoelastic surfaces has been developed based on a Greenwood-Williamson roughness distribution. For that purpose a simplified model has been proposed for the adhesive contact of viscoelastic asperities, assuming instantaneous loading and constant pull-out velocity. It retains the full phenomenology of the viscoelastic contact including stress relaxation inside the contact zone and creep in the cohesive zone.

For elastic surfaces, it is well known that increasing roughness rapidly suppresses adhesion. Our calculations give a quantitative assessment of how efficiently viscoelasticity restores adhesion on rough surfaces through the interplay between contact stress relaxation and cohesive zone creep. The phenomenon 
operates in a wide range of pull-out velocities.

\section{Acknowledgements}

We thank I. Sharma for numerous suggestions.

\section{Appendix - Computation of the total force}

The total force writes as [15]:

$$
P(t)=4 \int_{0}^{+\infty} g(r, t) d r
$$

The contribution of the cohesive zone is of higher order in $\epsilon$ (cohesive zone size) and is neglected, which is typical for large $\lambda$. Then Eq. 46 becomes $P(t)=$ $4 \int_{0}^{a(t)} g(r, t) d r$ and Eq. 13 becomes

$$
g(r, t)=\int_{t_{a-}(a(t))}^{t} d \tau \psi(t-\tau) \frac{d}{d \tau} \theta(r, \tau)
$$

with $\theta(r, \tau)=\delta(\tau)-\delta_{0}(r)$ which is Eq. 4. Inserting Eq. 47 into 46 and inverting the order of integration (cf also Ref. [15], section 3.2.4) results in Eq. 18,

\subsection{During the stick period}

During the stick period by assumption $a(t)=a_{0}$ is constant and $\min (a(t), a(\tau))=$ $a_{0}$. In addition for a sphere of radius $R$ loaded as specified in section 2.2.1, combining Eqs. 4 and 24

$$
\theta(r, \tau)=\left(\delta_{0}+\tau \dot{\delta}-r^{2} / R\right) \Theta(\tau)
$$

Then Eq. 18 gives:

$$
\begin{aligned}
& P_{1}(t)=4 \psi(t) \int_{0}^{a_{0}} d r\left(\delta_{0}-\frac{r^{2}}{R}\right)+4 a_{0} \dot{\delta} \int_{0}^{t} d \tau \psi(t-\tau) \\
& P_{1}(t)=4 \psi(t)\left(a_{0} \delta_{0}-\frac{a_{0}^{3}}{3 R}\right)+4 a_{0} \dot{\delta} \int_{0}^{t} d \tau \psi(t-\tau)
\end{aligned}
$$

where $P_{1}$ denotes the force during the stick period. The initial penetration $\delta_{0}$ is given by the double Hertz model and writes:

$$
\delta_{0}=\frac{a_{0}^{2}}{R}-\frac{\sigma_{0} \pi \sqrt{a_{0} \epsilon_{0}}}{\sqrt{2} E^{*}} .
$$

This last equation, in combination with the previous one, gives Eq. 25. 


\subsection{Decreasing contact radius}

By assumption the contact radius is constant or monotonously decreasing from $\tau=0$ so that $\min (a(t), a(\tau))=a(t)$ and Eq. 18 directly results in Eq. 31 . 


\section{References}

[1] Juang Y. J.; Bruer D.; Lee L. J.; Koelling K. W.; Srinivasan N.; Drummond C. H.; Wong B. C. J. Appl. Polym. Sci 2001, 80, 521.

[2] Zosel, A. J. Adhesion Sci. Technol. 1997, 11, 1447.

[3] Gay, C.; Leibler L. Phys. Rev. Lett. 1999, 82, 936.

[4] Pech J.; Berthome G.; Jeymond M.; Eustathopoulos N. Glass Sci. Technol. 2005, 78, 54 .

[5] Fuller, K.; Tabor, D. Proc. Roy. Soc. A 1975, 345, 327.

[6] Ting, T. C. T. J. Appl. Mech 1966, 33, 845.

[7] Schapery, R. A. Int J Fract 1975, 3, 369.

[8] Schapery, R. A. Int J Fract 1975, 11, 549.

[9] Greenwood, J. A.; Johnson, K. L. Phil. Mag. 1981, 43, 697.

[10] Greenwood, J. A. J. phys. D, Appl. phys. 2004, 37, 2557.

[11] Hui, C. Y.; Baney, J. M.; Kramer, E. J. Langmuir 1998, 14, 6570.

[12] Lin, Y. Y.; Hui, C. Y.; Baney, J. M. J. phys., D, Appl. phys. 1999, 32, 2250.

[13] Hui, C. Y.; Lin, Y. Y.; Baney, J. M. J. polym. sci., Part B, Polym. phys 2000, 38, 1485 .

[14] Barthel, E.; Haiat, G. Langmuir 2002, 18, 9362.

[15] Haiat, G.; Phan Huy, M. C.; Barthel, E. J. Mech. Phys. Sol. 2003, 51, 69.

[16] Barthel, E.; Haiat, G. J. Adhesion 2004, 80, 1.

[17] Schapery, R. A. Int. J. Fract. 1989, 39, 163.

[18] Lakes, R. S. and Wineman A. J. Elasticity 2006, 85, 45.

[19] Barthel, E.; Perriot, A. J. Phys. D 2007, 40, 1059.

[20] Barthel, E. J. Colloid Interface Sci. 1998, $200,7$.

[21] Greenwood, J. A.; Johnson, K. L. J. Phys. D: Appl. Phys. 1998, 31, 3279.

[22] Maugis, D. J. Colloid Interface Sci 1992, 150, 243.

[23] Greenwood, J.; Williamson, J. Proc. Roy. Soc. A 1966, 295, 300. 
[24] Greenwood, J. A.; Johnson, K. L. J. Colloid Interface Sci 2006, 296, 284.

[25] Maugis, D. J. Adhes. Sci. Technol. 1996, 10, 161.

\section{Captions and Figures}

Fig. 1. Schematics of the viscoelastic adhesive contact showing the gap between the surfaces and the normal surface stress distribution. Special emphasis is given to the cohesive zone where the attractive interactions operate across the gap between surfaces.

Fig. 2: Schematics of the relation between $g(a)$ - proportional to the stress intensity factor - and the crack velocity (after [14]).

Fig. 3: Prescribed penetration as a function of time $(\delta(t))$ and resulting contact radius history $(a(t))$. The constant radius approximation proposed in the present model is also shown as $a_{\text {appro }}(t)$. The end of the stick period is $t_{f}$.

Fig. 4: Single asperity force plot for different values of the pull-out velocity (from $\tilde{\dot{\delta}}=-0.01$ to $\tilde{\dot{\delta}}=-1$ ) and for the elastic limit (JKR) with the relaxed modulus.

Fig. 5: Single asperity force plot for different values of the pull-out velocity (from $\tilde{\dot{\delta}}=-1$ to $\tilde{\dot{\delta}}=-50$ ) and for the elastic limit (JKR) with the instantaneous modulus.

Fig. 6. Force distribution during adhesion rupture on a rough surface showing the contributions from asperities in the stick period and asperities with receding contact radius. The proportion of asperities in contact is shown on the right hand axis.

Fig. 7) Force plots for rough surfaces with increasing roughness. Here $\delta_{0}=5$ and $\dot{\delta}=-1$.

Fig. 8. Force plots for a rough surface with $\sigma_{s}=2$ for increasing pull-out velocity at low velocities. Also shown is the elastic limit for the relaxed modulus.

Fig. 9. Force plots for a rough surface with $\sigma_{s}=2$ for increasing pull-out velocity at high velocities. Also shown is the elastic limit for the instantaneous modulus.

Fig. 10: Pull-out force enhancement over elastic case as a function of pull-out velocity for an isolated asperity and for a rough surface with $\sigma_{s}=2$. 


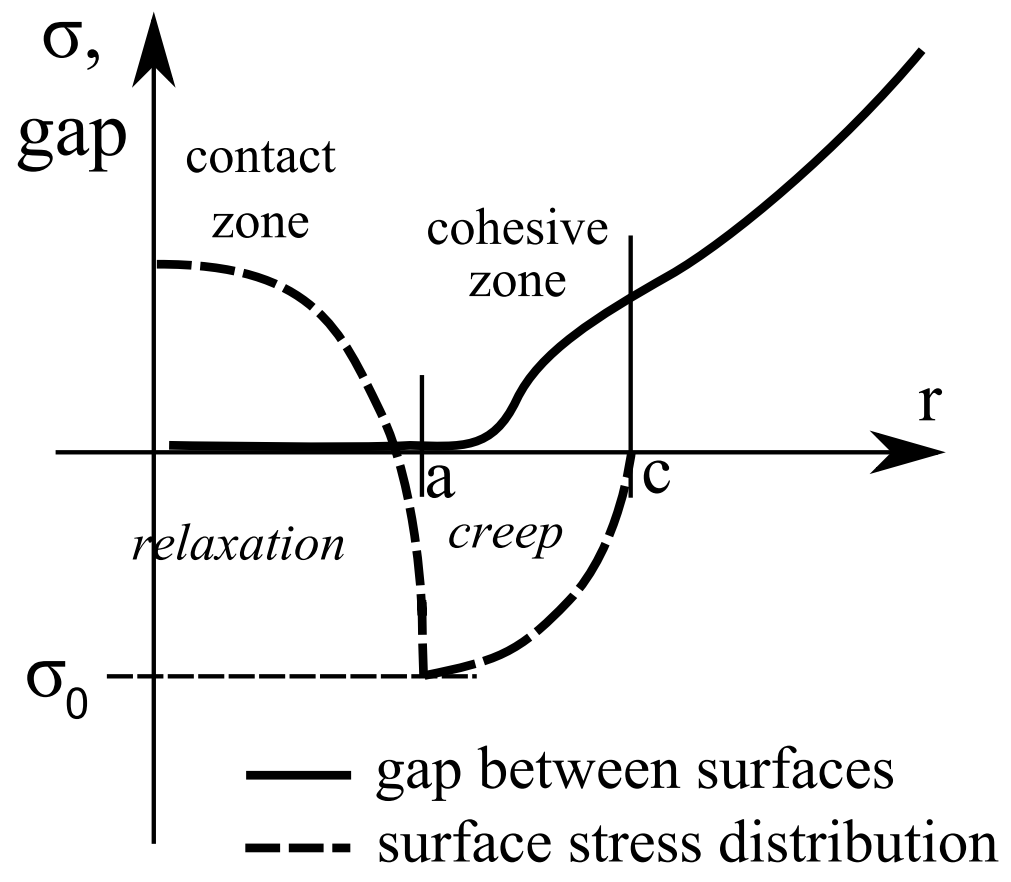

Figure 1:

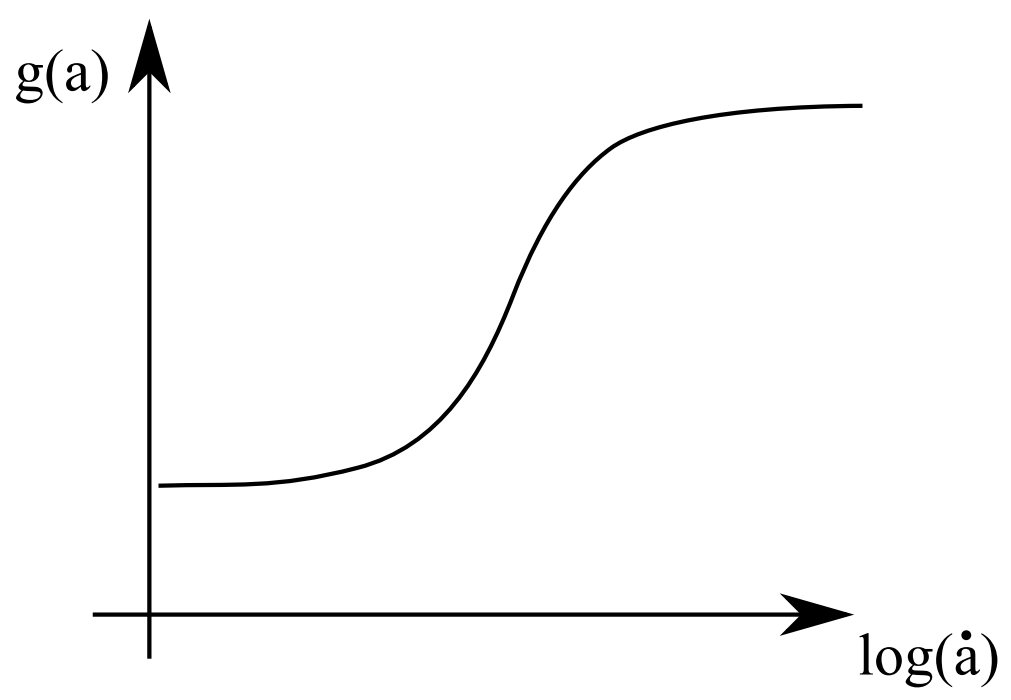

Figure 2: 


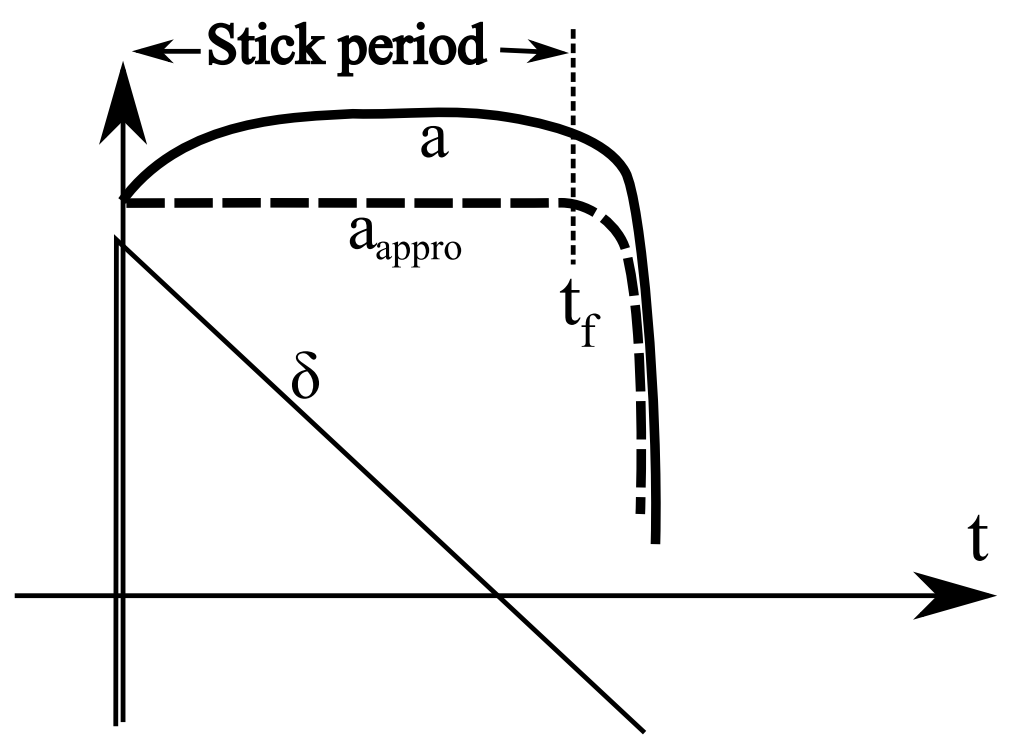

Figure 3:

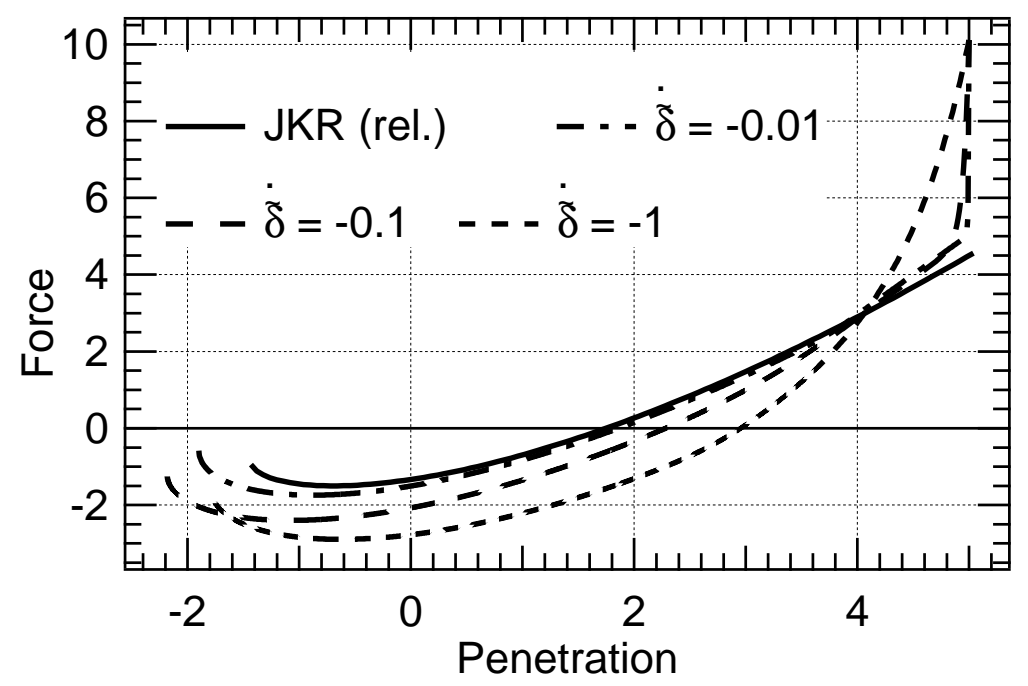

Figure 4: 


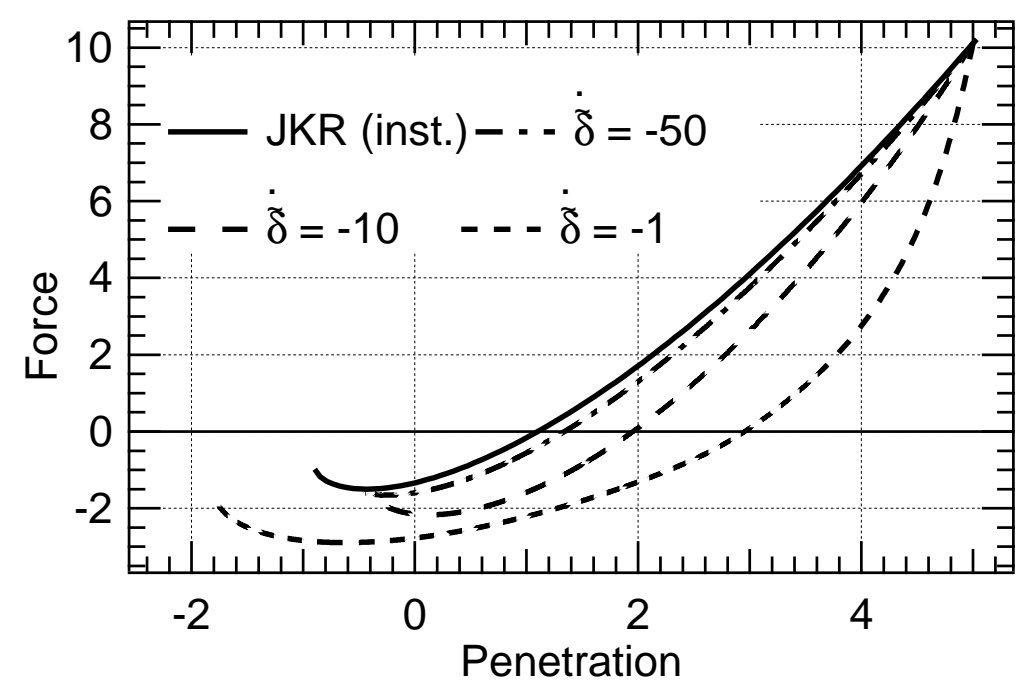

Figure 5:

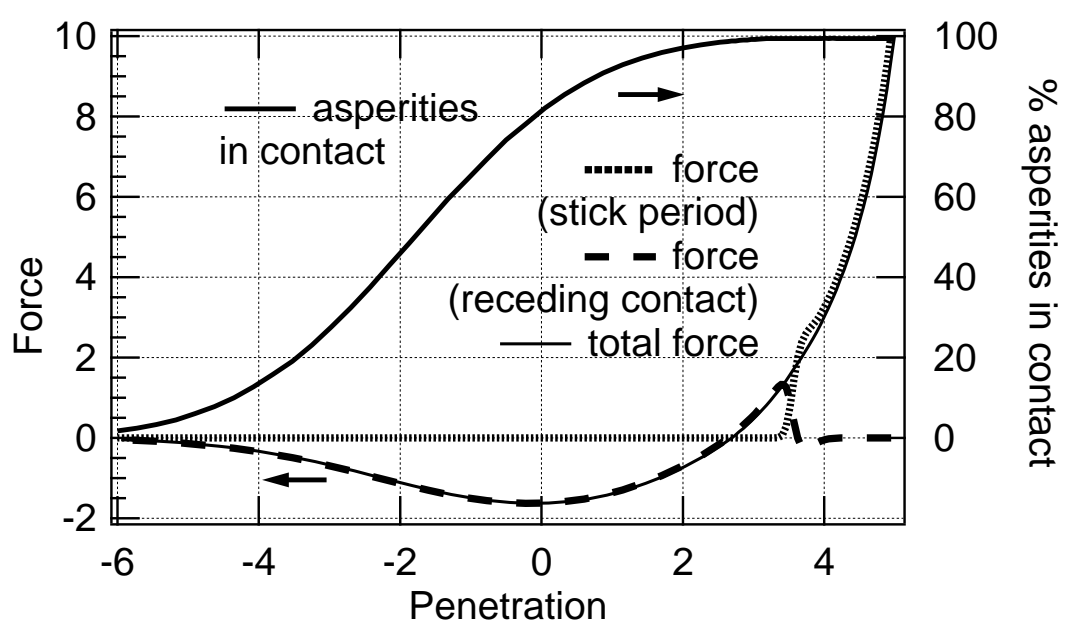

Figure 6: 


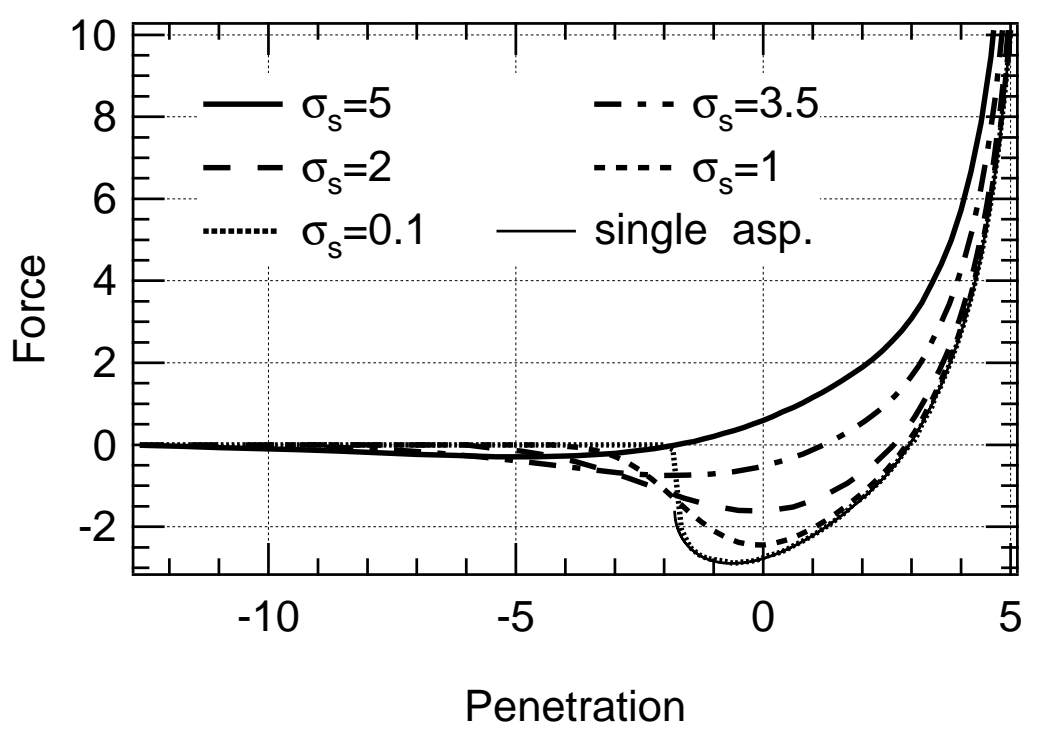

Figure 7:

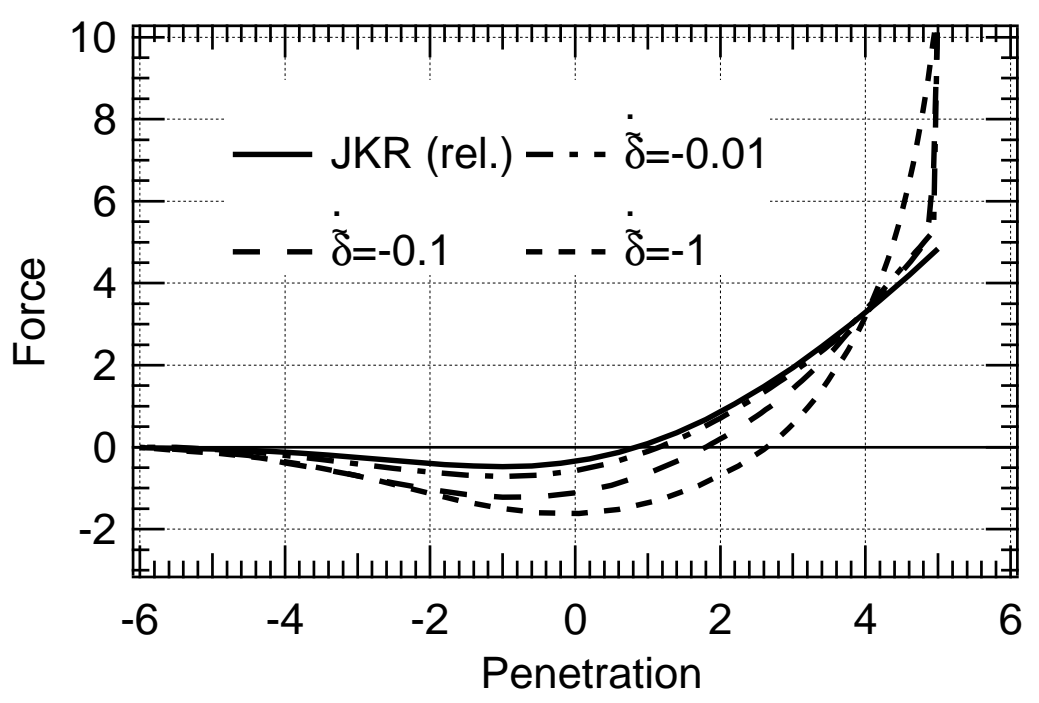

Figure 8: 


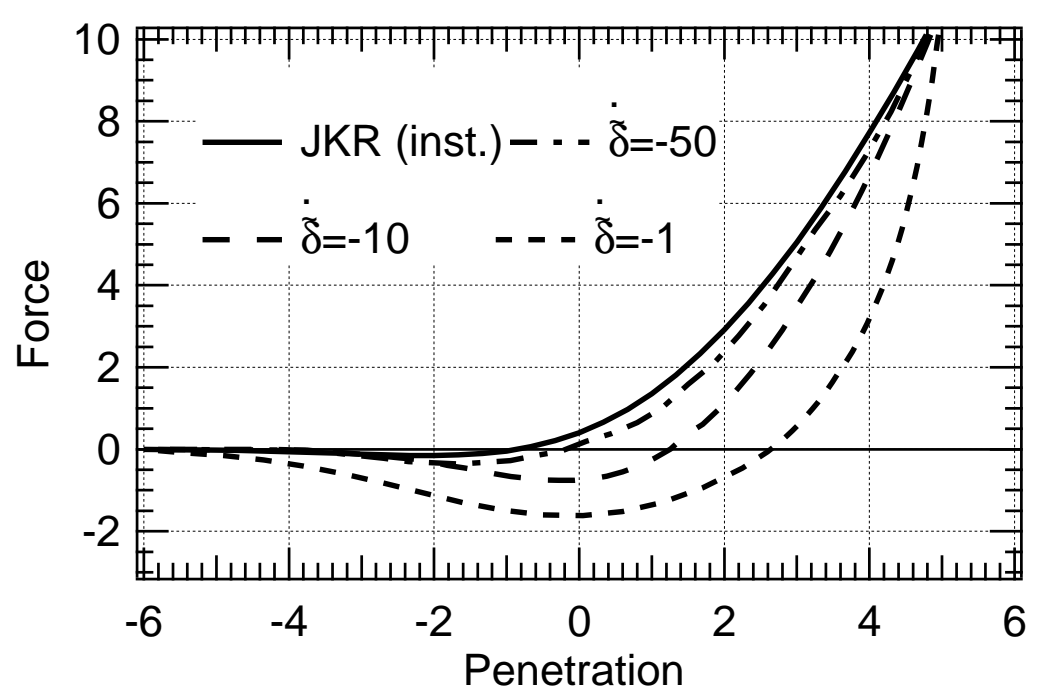

Figure 9:

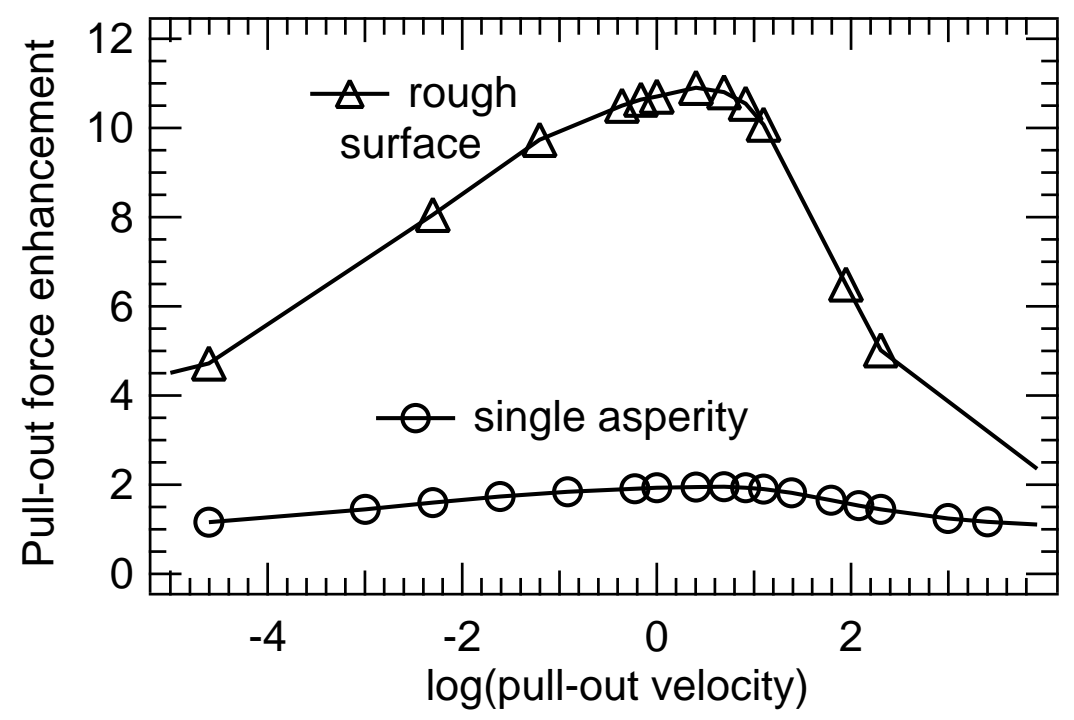

Figure 10: 


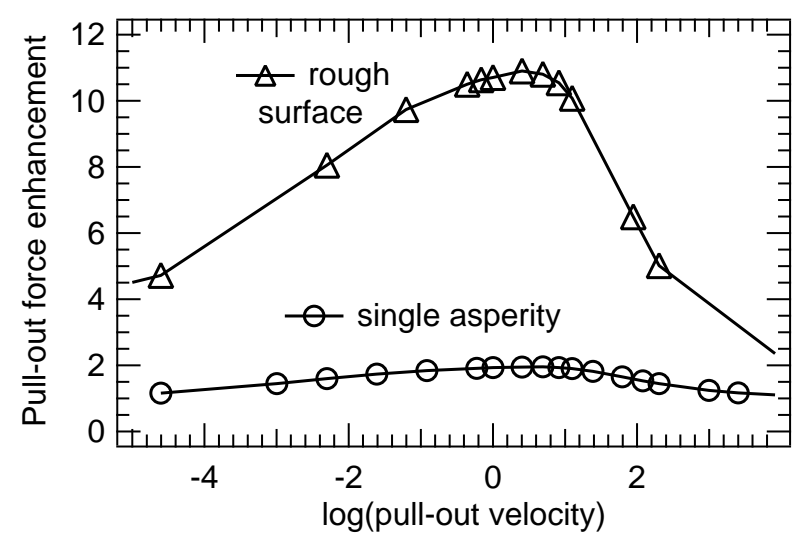

Figure 11: Table of Contents Graphic 